\title{
Patient Experience of Long-Standing Recovery Consequent to Surgical Management of Closed Fractures of Ankle: A Qualitative Study of Patient- Reported Outcomes in the Community
}

\author{
Sunil S Nikose ${ }^{1 *}$, Devashree Nikose ${ }^{2}$, Aditya Kekatpure $^{3}$, Kiran Saoji ${ }^{4}$, Sandeep Shrivastava ${ }^{5}$, Shashank \\ Jain $^{6}$ \\ ${ }^{1}$ Department of orthopedic surgery and Director of Centre of Excellence for Medical simulation studies (School of Virtual Learning), Datta Meghe Institute of \\ Medical Sciences, India
}

${ }^{2}$ Medical Doctor, NKP Salve Institute of Medical Sciences, Nagpur, India

${ }^{3}$ Department of orthopaedic surgery, Datta Meghe Institute of Medical Sciences, India

${ }^{4}$ Department of orthopaedic surgery, Datta Meghe Institute of Medical Sciences, India

${ }^{5}$ Director of orthopaedic surgery, Datta Meghe Institute of Medical Sciences, India

${ }^{6}$ Department of orthopaedic surgery, Datta Meghe Institute of Medical Sciences, India

*Corresponding author: Sunil S Nikose, Department of orthopaedic surgery and Director of Centre of Excellence for Medical simulation studies (School of Virtual Learning), Datta Meghe Institute of Medical Sciences, Sawangi -Meghe, Wardha, India
Received Date: August 17, 2020

Published Date: September 08, 2020

Abstract

Objective \& Aim: Ankle fractures and associated ligamentous injuries are common injuries of the load bearing articulation and result in unpredictable outcomes along with excessive prevalence of painful arthritis of ankle in the long term. With increasing age and co-morbidities, there are more complications and less than optimal outcomes despite optimal osteosynthesis related to this injury. There is little evidence concerning the long-standing outcome regarding these injuries. Hence the nucleus of this research, analysis has been having a greater perception of patient's expectations and recovery outcome in long term after ankle fractures along with identifying risk of poor ankle function in operated patients

Methods: We retrospectively analysed a group of 774 adults sustaining a closed ankle fractures treated with surgery. The outcomes were assessed using the Patient Reported Outcomes Measurement Information System for physical function (PROMIS- PF), PROMIS for pain interference (PROMIS-PI), Olerud-Molander Ankle Score (OMAS), Linear Analogue Scale (LAS) individually graded function of ankle. Short-Form 36 (SF-36) and radiographic examination at six weeks, six months, one and two-year follow-up.

Result: The whole cohorts were surgically managed according to the AO/ASIF practices. PROMIS measurements exhibited a higher ASA status $(\mathrm{P}=0.004)$, high body mass index (BMI) $(\mathrm{P}=0.009)$, and increasing age $(\mathrm{P}=0.03)$ turned out to be subjectively affiliated with diminished PROMIS$\mathrm{PF}$ assessments whereas a greater American Society of Anaesthesiologists (ASA) class $(\mathrm{P}=0.002)$ and reduced $\mathrm{BMI}(\mathrm{P}=0.016)$ and associated osteoporosis (Lower Bone Mass Density-BMD) were autonomously linked with increased PROMIS-PI grades. The follow-up occurred 6.7 (SD \pm 0.82 ) weeks, 6.8 ( $\mathrm{SD} \pm 0.96)$ months, $12.8(\mathrm{SD} \pm 0.85)$ months, 25.5 ( $\mathrm{SD} \pm 1.39$ ) months subsequent to surgery and the intermediate OMAS was 58.0 (IQR 31.55), 60.0 (IQR 32.65), 75.0 (IQR 33.25) and 77.26 (IQR 32.26) respectively. In the arenas of PROMIS-PF the responsive emotions, sociability, pain physique, intellectual health, energetic the health was notably inferior grades during the initial SF-36 as related to the subsequent SF-36. Conclusion: We found that most patients perform well within a year after surgically treated ankle fractures but continue to experience minor pain along with restricted clinical activeness. The patients who had restricted PF were those with increased BMI, higher ASA grade and old age and the patients who had increased pain interference (PI) and limitations were the ones with lower BMI, increased ASA grade and lower BMD. 
Keywords: Ankle fractures; Patient Reported Outcomes Measurement Information System (PROMIS); Physical Function (PF); Pain Interference (PI); Short-Form 36 (SF-36); Olerud-Molander Ankle Score (OMAS); Linear Analogue Scale (LAS)

Abbreviations: AO-Arbeitsgemeinschaft für Osteosynthesefragen; ASIF-Association for the study of internal fixation; PROMIS-Patient Reported Outcomes Measurement Information System; PROMIS -PF-Patient Reported Outcomes Measurement Information System for physical function; PROMIS-PI Patient Reported Outcomes Measurement Information System for pain interference; OMAS-Olerud-Molander Ankle Score; LASLinear Analogue Scale; SF-36-Short-Form 36; ASA-American Society of Anaesthesiologists; BMD -Bone mass density, BMI -Body mass Index; IQR -Interquartile range; ORIF -Open reduction and Internal fixation; 3D CT-Three dimensional computed tomography; SD-Standard Deviation; CATcomputerized adaptive test; NIH-National Institute of Health; QOL-Quality of life; ADL -Activities of daily living, LOS-Length of stay; GH-General Health; SF -Social function; PF-Physical function; RP -Role restriction caused by physical problem; MH-Mental health; BP-Bodily pain; VT-Vitality, SPSS $®-S o f t w a r e$ for statistical analysis (Version 11.5); PCC-Pearson correlation coefficient; WSR-Wilcoxon Signed- Rank, CM-centimetres

\section{Introduction}

Ankle fractures have been amongst the most widely recognized lower limb injuries treated by orthopaedic surgeons and most orthopedicians treat unstable ankle fractures as a surgical treatment $[1,2]$. Fractures in the ankle often lead to disabling ankle pain, muscle weakness, limited range of movements (ROM) in the weight-bearing ankle mortise, and difficulty in walking and climbing stairs [3-6].

Over the last few decades, the timeline revealed a surge in ankle injuries, especially among younger active individuals in addition to the geriatric population due to osteoporosis [7]. Ankle fractures operative procedure comprises open reduction and internal fixation (ORIF) in conjunction with meticulous postoperative restoration programme aimed at returning to pre-injury levels of activities $[8,9]$. The extent of ankle fractures has been traditionally described and subdivided into three subgroups namely - unimalleolar, bimalleolar and trimalleolar fractures. There is an increased threat of posttraumatic incongruity of ankle joint leading to painful ankle with high-energy injuries and fracture-dislocation of the ankle. Whereas the outcome of ORIF of ankle fractures regarding isolated lower fibular fractures, both malleoli fractures and trimalleolar fractures seemed to be reported more often; the patient- focused and confirmed clinically practical consequences of these injuries have been less often reported and the literature is scarce [10-12]. Some studies have shown contradictory findings while investigating the variations in functional outcomes between the severity groups and some concluded that a classification of the severity of fractures is a good predictor of functional outcomes after surgery [11,12]. There is no single predictor of ankle function after injuries and therefore the literature is flooded with numerous ankle scores, each claiming the advantage over the other. The universal factor agreed upon for good prognosis is a clinically pain-free ankle along with clinic radiologically congruent ankle which should be horizontal with stable tibiotalar and inferior tibiofibular articulation.

Considering these inadequacies in mind we designed the research to have a more detailed understanding of function, patient's expectations and recovery outcome during the long- term two years after surgical care of ankle fractures.

\section{Methods}

Subjects -A cohort of 774 adults sustaining a closed ankle fractures managed with surgery represented the retrospective observational research in patients treated from June 2015 to May 2017 which was carried out at the orthopaedic trauma unit of a university hospital. Among them 596 were men and 178 were women. Patients who were treated operatively according to Arbeitsgemeinschaft für Osteosynthesefragen (AO) / Association for the study of internal fixation (ASIF) methods with open reduction were included in the study along with a preoperative three dimensional computed tomography (3D CT) for intraarticular fractures and the syndesmotic injuries were treated with the repair of syndesmoses. Patients' demographic characteristics, mechanism of injury and injury pattern was extracted via an electronic medical system (EMS) scrutiny. Univariate and multivariate retrogression prototypes were established for resolution of self-determining prognosticators of PROMIS-PF and PROMIS-PI during the follow-up. Patients with neurological impairments like cognitive impairments, additional leg injury apart from ankle fractures, multiple trauma patients, stable ankle fractures requiring non-operative management, Pilon and its variants, Maisonneuve fractures, neuropathic ankle, previous surgery at ankle, fractures older than two weeks and patients whose assessment would be difficult due to multivariable factors or any condition preventing gait -analysis and completion of self-evaluation sampling were excluded. The follow up and radiographic examination was done at six weeks, six months and one and two years.

\section{Design of retrospective observational study}

All included subjects were briefed regarding research methodology in addition to objective conventions, any recognised compromises, and the consent obtained and sanctioned by the ethical committee of the institute.

\section{Outcome methods}

Patient-Reported Outcomes Measurement Information System of Physical Function (PROMIS - PF) and pain interference (PROMISPI) measurement. 
Our cohort included 774 consenting adults were evaluated telephonically, personally in clinic or via electronic mail (email). Patients were evaluated using PROMIS physical function v1.2 (PROMIS- PF) and PROMIS pain interference v1.1 (PROMIS -PI) via PROMIS analysed by computerized adaptive tests (CATs) forms. PROMIS was devised by the National Institute of Health (NIH) and is used to keep track of social, mental and physical health [13]. PROMIS-PF processes the capacity to accomplish actions requiring physical activity such as manoeuvrability, skilfulness, and the essential activity of the head and cervical spine along with activities of daily living (ADL) with a superior PROMIS-PF point being indicative of a positive objective while the PROMIS-PI assesses the painful consequences operational for wellbeing of the patient and quality of life (QOL), hampering the patients bodily functions, intellectual, and societal liveliness. A greater PROMIS- PI score is suggestive of the painful impediment of activity of daily living (ADL). Both these PROMIS $®$ points are assimilated ranging from zero to hundred (0-100) and a score of 50 is used as a reference for the general population.

\section{Fracture characteristics and treatment}

Standardized protocols record information such as injured side, mode and mechanism of injury, fracture pattern, surgical modes, length of stay (LOS) in hospital, non-weight bearing threshold, co-morbidities and complications. The ORIF interventions were conducted as per the AO/ASIF methods. The key results were assessed at six weeks, six months, one year and two years of followup. The foremost assessment was the Olerud-Molander Ankle Score (OMAS).

\section{Olerud-Molander Ankle Score (OMAS)}

The Olerud-Molander Ankle Score (OMAS) exists as a tool consisting of cross-examination questions of the patientadministered by himself. It is a functional assessment tool from null character (zero) to 100 in which the impairment is inversely proportional to the score which is predicated on nine different parameters such as pain in the ankle, ankle stiffness, swollen ankle, negotiating staircase, sprinting or jogging, hopping, ability to squat, daily living supports and activities. The OMAS is highly reliable, reproducible and is often acquainted with subjective assessment of ankles after the injuries to ankle [14].

\section{Linear Analogue Scale (LAS)}

LAS acts as a subjective ankle function with the ends marked "worst possible function" and a "perfectly normal function" functioning with 15 centimetres $(\mathrm{cm})$ spread out on the linear analogue scale (LAS) measured as a proportion of "perfectly normal function" ranked to $100 \%$ [14]. LAS maintains its comparison at odds with the OMAS in ankle injuries and fractures that have been surgically treated, and both scores have established a parallel correlation

\section{Self-rated ankle function}

It is a numerical evaluation of the patient's function pertaining to ankle from one to five and rated as 'very good' = One, 'good' = Two, 'fair'=Three, 'poor' =Four and 'very poor'= Five.

\section{Short-Form 36}

The Short-Form 36 (SF-36) functions as a standardized set of queries where the patient has control over the response outline relating to QOL within the correlation of health. This instrument evaluates eight wellness indexes using measures of general health $(\mathrm{GH})$, social function (SF), physical function (PF), role restriction caused by physical problems (RP), mental health (MH), bodily pain (BP), vitality (VT), and role impediment attributable to emotional problems (RE). Further stratification has a score of null to hundred $(0-100)$ with a stunted tally summarizing the poorer state of healthiness and vice versa [15]. The SF-36 was established for the Swedish population and standardizing results were published keeping in mind the general health of the population. However, no studies have been carried out evaluating the dependability and sustainability for SF-36 use in cohorts sustaining fractures of the ankle.

\section{Statistical Interpretation}

Utilizing the SPSS $®$ statistics (11.5 version) software anatomization was carried out employing univariate and multivariate retrogression determinants. Uninterrupted parameters were established for underlying parametric and non-parametric assumptions statistics and defined and analysed as needed. The Wilcoxon Signed-Rank (WSR) assessment was accustomed to evaluating variations amongst six months, one year and two-year follow-up with respect to OMAS, LAS and individually graded function of ankle, while the Mann-Whitney U-test became the gold standard to examine gender disparities specifically to that of age, OMAS, LAS and individually graded ankle function. In each of these eight SF-36 domains, a Student t-test, which was onetailed and double barrelled was executed for each patient's SF-36 cross-examination due to a simple reason that it is not expected for the scores to fall due to this test, along with a p-value of $<0.05$ was deemed considerable. Pearson correlation coefficients (PCC) towards absolute determinants was adapted for establishing risk stratification associated with statistically appreciable PROMIS- PF and PROMIS-PI scores.

\section{Observations and Result}

Amongst the total 774 patients, 596 (77.01\%) were male and 178 (22.99\%) were female. Age distribution variables, mode of trauma, laterality is characterized in (Table 1 ).

\section{Fracture pattern and PROMIS- PF and PROMIS - PI measurement}

Based on the AO/OTA classification of ankle injuries fractures were divided into 44-A (Infrasyndysmotic) -53.88\%; 44-B 
(transsyndysmotic) - 37.47\% and 44-C (suprasyndysmotic) $8.65 \%$ The mean age, risk factors like Diabetes Mellitus, smoking and ASA grades were recorded. The mean PROMIS- PF score was 52.97 (SD = 9.45) in addition to the PROMIS - PI indicative score of 47.03 ( $S D=7.89$ ). It turned out to be of no relevance disparity between PROMIS-PF or PROMIS-PI result considering different ankle fracture classifications and neither the syndesmotic injury (PF -P = 0.45 and $\mathrm{PI}-\mathrm{P}=0.77$ ) nor the posterior malleolar fracture (PF $-\mathrm{P}=$ 0.23 and $\mathrm{PI}-\mathrm{P}=0.55$ ) or the medial malleolar fracture ( $\mathrm{PF}-\mathrm{P}=0.13$ and PI $-\mathrm{P}=0.38$ ) had any significant difference and is described in Table 2. Female gender, increasing age, diabetes mellitus (DM), and higher BMI, in addition to higher ASA status, turned out to be interconnected with lower PROMIS-PF scores as shown in Table 2.
However, tobacco smoking in conjunction with fracture dislocation of ankle had no connection with considerable dissimilarity in regard to PROMIS-PF results. Moreover, the presence of DM, greater ASA physical status, and lesser BMI correlated with greater PROMISPI results but the gender, age, tobacco use, fracture-dislocation of ankle remained insignificant in consideration of the PROMIS-PI results. Subsequent to adjustments in control and disconcerting components, only the higher ASA status $(\mathrm{P}=0.004)$, elevated BMI $(\mathrm{P}=0.009)$, and increasing age $(\mathrm{P}=0.03)$ demonstrated reduced PROMIS-PF results by multivariable retrogression whereas, high ASA physical status $(\mathrm{P}=0.002)$ and low BMI $(\mathrm{P}=0.016)$ autonomously demonstrated interconnected inflated PROMIS-PI results (Table 2).

Table 1: Age, gender and wound complications in treated patients of closed ankle fractures.

\begin{tabular}{|c|c|c|}
\hline Variables & Parameters & Number of Patients and Percentage (\%) \\
\hline \multirow{2}{*}{ Gender } & Male & $596(77.01 \%)$ \\
\hline & Female & $178(22.99 \%)$ \\
\hline \multirow{2}{*}{ Injured site } & Right & $403(52.06 \%)$ \\
\hline & Left & $371(47.93 \%)$ \\
\hline \multirow{6}{*}{ Age group } & 18 -30 years; Mean $26.36 \pm 4.23$ & $172(22.22 \%)$ \\
\hline & $31-40$ years; Mean $35.75 \pm 5.62$ & $160(20.67 \%)$ \\
\hline & $41-50$ years; Mean $47.56 \pm 5.36$ & $141(18.28 \%)$ \\
\hline & $51-60$ years; Mean $56.18 \pm 6.21$ & $130(16.79 \%)$ \\
\hline & $61-70$ years; Mean $66.66 \pm 5.89$ & $92(11.88 \%)$ \\
\hline & 71 years and above; Mean $73.55 \pm 3.98$ & $79(10.20 \%)$ \\
\hline
\end{tabular}

Table 2: Ankle fracture characteristics in terms of PROMIS- PF and PROMIS-PI.

\begin{tabular}{|c|c|c|c|}
\hline \multirow{2}{*}{ Variables } & \multirow{2}{*}{ Percentage of Patients } & \multicolumn{2}{|c|}{ Significance (P Value) } \\
\hline & & Physical Function PROMIS-PF & Pain Interference PROMIS-PI \\
\hline \multirow{3}{*}{$\begin{array}{c}\text { AO/OTA classification } 44 \text {-A -Infrasyn- } \\
\text { dysmotic } 44 \text {-B - Transsyndysmotic } 44-\mathrm{C} \\
\text { - Suprasyndysmotic }\end{array}$} & $53.88 \%$ & \multirow{3}{*}{0.36} & \multirow{3}{*}{0.25} \\
\hline & $37.47 \%$ & & \\
\hline & $8.65 \%$ & & \\
\hline Syndesmosis injuries & $45.21 \%$ & 0.45 & 0.77 \\
\hline Medial Malleolus & $38.75 \%$ & 0.13 & 0.38 \\
\hline Posterior malleolus & $27.90 \%$ & 0.23 & 0.55 \\
\hline Combined fracture-dislocation pattern & $36.15 \%$ & 0.78 & 0.79 \\
\hline Low Energy injury & $33.33 \%$ & \multirow{2}{*}{0.29} & \multirow{2}{*}{0.22} \\
\hline High Energy Injury & $66.67 \%$ & & \\
\hline \multicolumn{4}{|c|}{ Risk Factors } \\
\hline Gender: Male & $77.01 \%$ & 0.03 & 0.56 \\
\hline Female & $22.99 \%$ & & \\
\hline Age & $51.01(18-82)^{a}$ & $<0.01$ & 0.86 \\
\hline Diabetes & $14.34 \%$ & 0.03 & 0.08 \\
\hline Smoking & $19.64 \%$ & 0.78 & 0.35 \\
\hline BMI & $29(18-45)^{\mathrm{a}}$ & $<0.01$ & $<0.01$ \\
\hline ASA Physical status Class - I & $50.39 \%$ & $<0.01$ & $<0.01$ \\
\hline ASA Physical status Class -II & $43.15 \%$ & & \\
\hline ASA Physical status Class- III & $6.45 \%$ & & \\
\hline
\end{tabular}


Patients were rehabilitated based on their injuries, age, pain tolerance, bone quality, quality of osteosynthesis, local wound complications and BMD. A large number of individuals were toe touch or non-weight bearing for initial one and half months during the post-operative phase : $643(83.07 \%)$ patients, whereas after 6 weeks there were still $140(18.08 \%)$ patients who were advised against full weight-bearing on their injured lower extremity.

\section{Olerud-Molander Ankle Score (OMAS)}

The initial follow up for functional assessment materialized at six weeks; 6.7 (SD 0.82) weeks post-surgery with the average OMAS being 58.0 (IQR 31.55) whereas the subsequent evaluation at 6.8 (SD 0.96) months post - surgery revealed an OMAS of 60.0 (IQR 32.65). The one year and two years follow up demonstrated an OMAS of 75.0 (IQR 33.25) and 77.26(IQR 32.26) respectively. The gender variability revealed no significant difference except that the women had significant improvement $(p=0.002$ and 0.003$)$ in subsequent follow up as depicted in Table 3.586 (75.71\%) subjects reported pain at six weeks, $422(54.52 \%)$ at six months and 302 (39.01\%) after a years' time and at two years while ambulating on irregular planes. Almost 60\% individuals' stated ambulatory oedema negotiating stairs and around $50 \%$ of patients declared ankle and foot stiffness in one year of follow up which barely got better in the last follow up.

\section{Linear Analogue Scale (LAS)}

The intermediary Linear Analogue Scale (LAS) at six weeks, half yearly, first year and second year had been70.0 (IQR 28.10), 73.0 (IQR 27.0), 83.0 (IQR 30.0) and 84.96 (IQR 29.96) respectively. There was a parallel association between OMAS and LAS which is depicted in Table 3.

\section{Self-rated ankle function}

The Intermediary self-rated ankle function in our total sample $(p=0.05)$ reduced gradually over a period of time from the initial to the final follow up and is depicted in (Table 3).

\section{Association apropos OMAS with Clinical Assessments}

The grouping with regards to individuals was based on ankle movements (dorsiflexion and plantarflexion), muscular strength of leg (circumference the difference of $\geq 1 \mathrm{~cm}$ ), along with maintenance of equilibrium on a single leg stance $(\leq$ one minute on the injured leg) and then correlated with OMAS which revealed that the lesser OMAS results are associated with inferior clinical assessments in the three parameters as stated above and is depicted in (Table 4).

Table 3: Outcomes from OMAS, LAS, self-rated ankle function at different duration after surgical treatment of ankle fractures.

\begin{tabular}{|c|c|c|c|c|c|c|}
\hline $\begin{array}{l}\text { Outcomes Analysis } \\
\text { of Different Scores }\end{array}$ & Gender (N) & $\begin{array}{l}\text { 6-Weeks Follow-Up } \\
\text { Median (IQR) }\end{array}$ & $\begin{array}{l}\text { 6-Month Follow-Up } \\
\text { Median (IQR) }\end{array}$ & $\begin{array}{l}\text { 12-Month Follow-Up } \\
\text { Median (IQR) }\end{array}$ & $\begin{array}{l}\text { 2-Year Follow-Up } \\
\text { Median (IQR) }\end{array}$ & P-Value \\
\hline \multirow{3}{*}{ OMAS $(0-100)$} & Female (178) & $52.0(36.19)$ & $56.0(37.09)$ & $68.0(30.22)$ & $70.0(35.22)$ & 0.005 \\
\hline & Male (596) & $62.5(36.31)$ & $65.5(37.37)$ & $80.0(28.75)$ & $81.03(34.26)$ & 0.161 \\
\hline & Total (774) & $58.0(31.55)$ & $60.0(32.65)$ & $75.0(33.25)$ & $77.26(32.26)$ & 0.003 \\
\hline \multirow{3}{*}{ LAS $(0-100)$} & Female (178) & $65.0(30.5)$ & $70.0(33.5)$ & $85.0(37.0)$ & $87.02(36.23)$ & 0.011 \\
\hline & Male (596) & $75.0(25.25)$ & $76.0(20.5)$ & $80.0(30.5)$ & $82.03(28.23)$ & 0.401 \\
\hline & Total (774) & $70.0(28.10)$ & $73.0(27.0)$ & $83.0(30.0)$ & $84.96(29.96)$ & 0.01 \\
\hline \multirow{3}{*}{$\begin{array}{l}\text { Self-rated ankle func- } \\
\quad \text { tion }(1-5)^{*}\end{array}$} & Female (178) & $3.0(1.0)$ & $3.0(1.0)$ & $2.0(1.5)$ & $2.0(1.5)$ & 0.06 \\
\hline & Male (596) & $3.0(1.0)$ & $3.0(1.0)$ & $2.0(1.0)$ & $2.0(1.0)$ & 0.5 \\
\hline & Total (774) & $3.0(1.0)$ & $3.0(1.0)$ & $2.0(2.0)$ & $2.0(2.0)$ & 0.05 \\
\hline
\end{tabular}

IQR = Interquartile range; *1 = Very good; 2 = good; 3 = fair; 4 = poor; 5 = very poor

Table 4: Comparing OMAS and clinical assessments based on different parameters: 1 . Ankle movements: 2 . Maintenance of equilibrium and 3. Muscle strength.

\begin{tabular}{|c|c|c|c|}
\hline Various Clinical Ankle Parameters & Number of Patients $(\mathrm{N}=774)$ & OMAS (Medium (Range)) & P Value \\
\hline Dorsiflexion $\geq 30^{\circ}$ & 424 & $80.0(40-100)$ & \multirow{2}{*}{0.04} \\
\hline Dorsiflexion $<30^{\circ}$ & 350 & $60.0(10-100)$ & \\
\hline Plantar flexion $\geq 45^{\circ}$ & 434 & $80.0(40-100)$ & \multirow{2}{*}{0.003} \\
\hline Plantar flexion $<45^{\circ}$ & 340 & $65.0(10-100)$ & \\
\hline Toes Rising $\geq 25$ & 441 & $85.0(10-100)$ & \multirow{2}{*}{0.08} \\
\hline Toes Rising $<25$ & 333 & $65.0(40-100)$ & \\
\hline Heel Rising $\geq 20$ & 502 & $85.0(10-100)$ & \multirow{2}{*}{0.05} \\
\hline Heel Rising $<20$ & 272 & $65.5(40-100)$ & \\
\hline Stability $\geq 60$ seconds & 429 & $85.0(10-100)$ & \multirow{2}{*}{0.009} \\
\hline Stability $<60$ seconds & 345 & $65.0(40-100)$ & \\
\hline Circumference difference: $1 \mathrm{~cm}$ & 325 & $85.5(45-100)$ & \multirow{2}{*}{0.04} \\
\hline Circumference difference $\geq 1 \mathrm{~cm}$ & 449 & $65.0(10-100)$ & \\
\hline
\end{tabular}




\section{Short-form 36 (SF-36)}

The first cross-examination by the SF-36 form initially was done at 6.9 weeks ( \pm SD 0.78 ; range, $5.2-11$ weeks) followed by 6.4 months ( \pm SD 0.65 ; range, $5.5-7.0$ months), 13.4 months $( \pm S D$
6.2; range, 11-14 months) and 25.6 months ( \pm SD 7.6; range, 2228 months). Most areas saw significant differences except for the general health (GH) status which is exhibited in (Figure 1)

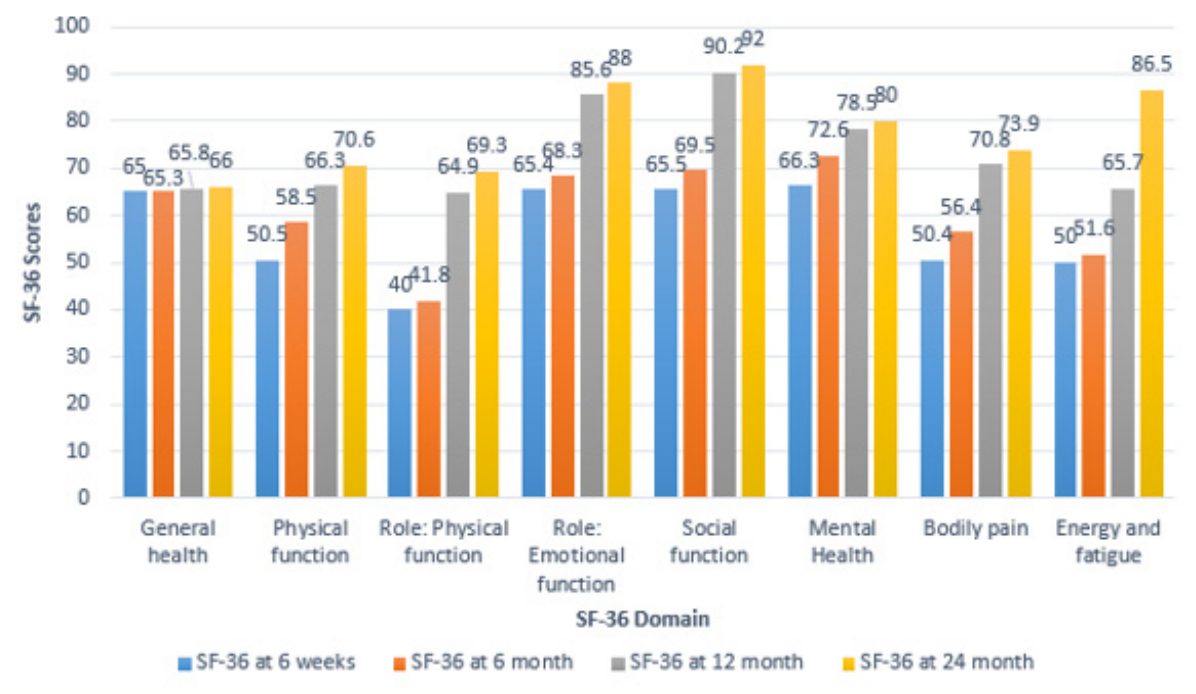

Figure 1: Comparison of SF-36 scores at 6 weeks, 6 month, 12 months and 24 months.

Radiological evaluation revealed that 702 (90.69\%) fractures demonstrated union by three months' time period. 56 (7.23\%) patients had a second surgical procedure within six months post-surgery attributable to problems associated with surgical hardware, while 12 (1.55\%) patients needed re-look debridement during the immediate postoperative phase. The average hospital length of stay (LOS) was six [spanning 2 - 50 (IQR 3.8)] days. At six months follow up 672 (86.82\%) had returned to the pre-injury level of living but with the persistence of mild pain [Visual Analogue Scale -VAS mean 2.8 (Range 2 - 5)], although 140 (18.08\%) patients required extended multiple admissions for hospital care for reasons related to local infection and hardware problems ranging from one and six weeks before they went back to their preinjury living. Moreover, there were nine (1.162\%) painless non-union of fibular malleolus and eleven (1.42\%) painful fibular malleolar non-union along with eight $(1.03 \%)$ medial malleolar non-unions requiring re-surgery. Fortunately, enough at a point of a year after trauma, there was no post-traumatic arthritis of the ankle. Two $(0.258 \%)$ individuals required early ankle arthrodesis for deep-seated ankle infection which could not be managed by repeated debridement and extended antibiotics. Various complications are illustrated in (Table 5).

Table 5: Postoperative complications of surgically treated closed ankle fractures.

\begin{tabular}{|c|c|c|}
\hline \multirow{2}{*}{ Nature of Complications } & Specific Complications & Number N= 774 \\
\hline \multirow{3}{*}{ Post-operative Wound Complications } & Superficial infection & $65(8.39 \%)$ \\
\cline { 2 - 3 } & Deep infection and skin necrosis & $12(1.55 \%)$ \\
\cline { 2 - 3 } & No wound complications & $697(90.05 \%)$ \\
\cline { 2 - 3 } Subsequent Surgery & Debridement & $12(1.55 \%)$ \\
\cline { 2 - 3 } & Hardware removal & $56(7.23 \%)$ \\
\cline { 2 - 3 } & Re-do osteosynthesis for failed ORIF & $28(3.617 \%)$ \\
\cline { 2 - 3 } & Ankle arthrodesis due to persistent deep-seated infection & $2(0.258 \%)$ \\
\hline
\end{tabular}

\section{Discussion}

Fractures in the ankle region are common musculoskeletal injuries which often requires operative stabilization [16]. While the clinical outcomes of non-displaced solitary malleolus might give acceptable results, the consensus is to achieve a congruous and stable ankle good enough for weight-bearing, avoidance of stiffness, elimination of osteodystrophy along with impediment of infection especially due to the superficial placement of both malleoli [17,18]. The practical status after sustaining ankle fractures have by all means measured nevertheless the conclusions 
are baffling and contradictory by various research groups $[19,20]$. An extended execution of analyses of subjects with malleoli was reported in such a manner where more than $50 \%$ of individuals experience discomfort, inflammation, oedema along with stiffness causing a proportion of numerous working incapacities [21]. Recently the focus on functional rehabilitation after orthopaedic interventions have increased where one of the research analysis regarding 141 ankle fracture individuals was followed for two years after operative stabilisation, furthermore, Lash et al observed that $77 \%$ had a positive and exceptional outcome based on OMAS score whereas around $21 \%$ of patients had minimalistic clinical impairments [20,22,23].

The sequence of the present research signals that most individuals carry on with complaints along with described practical restrictions after a year of their surgical management. While $82 \%$ of our patients were totally physically active prior to the trauma around $50 \%$ or less returned to their pre-injury status at the end of one year, though the individualized ankle function considered by OMAS, LAS, together with the self-rated ankle function, improved over the total sample between the four follow-ups similar to the findings of Nilsson et al in 2007 [24].

Earlier research in younger cohorts displayed improved OMAS scoring after a year of injury in comparison to our research. Hedström et al correlated fibular malleolar fractures one year after injury considering a midpoint of 42 years (range $16-71$ years) along with establishing that a mean OMAS score post-injury is 88 points [25]. Lehtonen et al analysed and correlated the age of patients in contrast to OMAS scoring system and reported a mean of 90 points for patients averaging 41 years of age whereas Van Laarhoven et al reported a median 95 points OMAS for patients averaging 36 years of age (range 17-77) with excellent scored function [26,27]. The results for different age groups were not presented in either of the studies. Tropp et al came out with an average OMAS score of 91 points in thirty individuals treated by ORIF with a standard age of 26 years [28]. Egol et al and Nilsson et al delineated that patients elder than 40 years are prognostically poor one year and three years' post-surgery and their findings are on par with our present research $[20,24]$.The above-mentioned outcomes reinforce the judgement regarding better results in young individuals as far as function is concerned and similar fracture patterns is nonhomogenous based on age.

Furthermore, the SF-36 assessment is a useful practical measure for post-fixation outcomes and is agreeably established for functional assessment thus enabling the activity of the patient to be correlated with community standard in numerous disorders and musculoskeletal injuries [29]. Ponzer et al studied various parametersafterinjuryanddemonstrated a considerably diminished SF-36 element tally compared to the population in a Scandinavian country where his patients had similar health conditions and the quality of life metrics declined dramatically relative to our patients accompanied by particular population standards demonstrating a similar age and a similar pattern of injuries [30]. Current research reveals no fundamental dissimilarity into SF-36 tally relative to the United States of America (USA). Guidelines in the concluding followup [31]. The SF-36 physical function (PF) arena is evaluated based on the acknowledgement of patient to certain restrictions in particular behaviours namely negotiating staircase, bathing, ambulation and leisure interest. Our statistics illustrate that patients continue to experience considerable improvement in function after four to six months nevertheless with some remaining physical incapacity. Additionally Diabetes mellitus, tobacco smoking, osteoporosis, high energy trauma, higher ASA grading and obesity has adverse association with final outcomes whereas educational status, AO/ OTA classification system, syndesmotic injuries, posterior and medial malleolar fractures and fracture-dislocation did not exhibit inverse correlation with operational impact during the long term. Determinants of PROMIS-PF diminished are higher ASA grading, geriatric population, and obesity whereas the heightened PROMISPI linked to increased pain encompassed those with lesser BMI, osteoporosis (Low BMD) and higher ASA grade which has the greatest adversary consequences during the postoperative phase.

\section{Conclusion}

A long-term analysis reveals that following surgically treated ankle fractures, most patients accomplish performance with most encountering minuscule soreness and discomfort and a small number will have limitations in useful pursuits. Most individuals had a substantial enhancement a year after surgery compared to within six months. Numerous factors delay the recovery at one year such as elderly age, higher ASA grading category, diabetes mellitus and in women population. The capacity to ascertain at-risk patients allows orthopaedic teams to transform the care pathway and optimize recuperation. More importantly, the patients and their caregivers should be informed throughout regarding the expectations of purposeful restoration after the injury.

\section{Disclosure of Potential Conflicts of Interest}

The authors declare that they have no conflict of interest.

\section{Ethics Approval and Consent to Participate}

The study protocol was approved by the Institutional ethical committee. All procedures performed in this study were in accordance with the ethical standards of the committee and with the 1964 Helsinki declaration and its later amendments or comparable ethical standards.

\section{Funding statement}

This research did not receive any specific grant from funding agencies in the public, commercial, or not-for-profit sectors.

\section{Data Availability}

The data used to support the findings of this study are available on reasonable request from the corresponding author (SN). The 
data are not publicly available due to the information that might compromise the privacy of research participants (Patients).

\section{Informed Consent}

All the patients provided written informed consent for the study format.

\section{Acknowledgement}

I would like to express my very great appreciation to Miss Bhagyashree Nikose for her valuable and constructive suggestions including direct technical assistance during the planning and development of this research article and she undertook this as a summer side project despite being busy with her academics. Her willingness to give her time so generously has been very much appreciated.

\section{References}

1. Lash N, Horne G, Fielden J, Devane P (2002) Ankle fractures: functional and lifestyle outcomes at 2 years. ANZ J Surg 72(10): 724-730.

2. Michelson JD (1995) Fractures about the ankle. J Bone Joint Surg Am 77: $142-152$.

3. Lin CW, Moseley AM, Herbert RD, Refshauge KM (2009) Pain and dorsiflexion range of motion predict short- and medium-term activity limitation in people receiving physiotherapy intervention after ankle fracture: an observational study. Aust J Physiother 55(1): 31-37.

4. Psatha M, Wu Z, Gammie FM, Ratkevicius A, Wackerhage $\mathrm{H}$, et al (2012) A longitudinal MRI study of muscle atrophy during lower leg immobilization following ankle fracture. J Magn Reson Imaging 35(3): 686-695.

5. Hancock MJ, Herbert RD, Stewart M (2005) Prediction of outcome after ankle fracture. J Orthop Sports Phys Ther 35(12): 786-792.

6. Beckenkamp PR, Lin CW, Chagpar S, Herbert RD, Van der Ploeg HP, et al. (2014) Prognosis of physical function following ankle fracture: a systematic review with meta-analysis. J Orthop Sports Phys Ther 44(11): 841-851.

7. Court-Brown CM, McBirnie J, Wilson G (1998) Adult ankle fractures-an increasing problem? Acta Orthop Scand 69(1):43-47.

8. Tile M, (1996) Fractures of the Ankle. In The Rationale of Operative Fracture Care. 2nd edition. (Eds) Schatzker J, Tile M. Berlin: Springer 523-561.

9. Donatto KC (2001) Ankle fractures and syndesmosis injuries. Orthop Clin North Am 32(1): 79-90.

10. Obremskey WT, Dirschl DR, Crowther JD, Craig WL 3rd, Driver RE, et al. (2002) Change over time of SF-36 functional outcomes for operatively treated unstable ankle fractures. J Orthop Trauma 16(1): 30-33.

11. Lash N, Horne G, Fielden J, Devane P (2002) Ankle fractures: functional and lifestyle outcomes at 2 years. ANZ J Surg 72(10): 724-730.

12. Day GA, Swanson CE, Hulcombe BG (2001) Operative treatment of ankle fractures: a minimum ten-year follow-up. Foot Ankle Int 22(22): 102106.

13. Michelson J, Solocoff D, Waldman B, Kendell K, Ahn U (1997) Ankle fractures. The Lauge-Hansen classification revisited. Clin Orthop Relat Res 345: 198-205.
14. Olerud C, Molander H (1984) A scoring scale for symptom evaluation after ankle fracture. Arch Orthop Trauma Surg 103(3): 190-194.

15. Ware JE, Sherbourne CD (1992) The MOS 36-item short-form health survey (SF-36). I. Conceptual framework and item selection. Med Care $30(6): 473-483$.

16. Phillips WA, Swartz HS, Keller CS, et al. (1985) A prospective randomized study of the management of severe ankle fractures. J Bone Joint Surg Am 67(1): 67-78.

17. Ponzer S, Nasell H, Bergman B (1999) Functional outcome and quality of life with patients with Type B ankle fractures: a two-year follow-up study. J Orthop Trauma 13(5): 363-368.

18. Van der Sluis CK, Eisma WH, Groothoff JW (1998) Long-term physical, psychological and social consequences of a fracture of the ankle. Injury 29(4): 227-280

19. Hancock MJ, Herbert RD, Stewart M (2005) Prediction of outcome after ankle fracture. J Orthop Sports Phys Ther 35(12): 786-792.

20. Egol KA, Tejwani NC, Walsh MG, Capla EL, Koval KJ (2006) Predictors of short-term functional outcome following ankle fracture surgery. J Bone Joint Surg Am 88(5): 974-979.

21. Segal G, Elbaz A, Parsi A, Heller Z, Palmanovich E, et al. (2014) Clinical outcomes following ankle fracture: a cross-sectional observational study. J Foot Ankle Res 7(1): 50

22. Obremskey WT, Dirschl DR, Crowther JD, Craig WL 3rd, Driver RE, et al. (2002) Change over time of SF-36 functional outcomes for operatively treated unstable ankle fractures. J Orthop Trauma 16(1): 30-33.

23. Lash N, Horne G, Fielden J, Devane P (2002) Ankle fractures: functional and lifestyle outcomes at 2 years. ANZ J Surg 72(10): 724-730.

24. Nilsson G, Jonsson K, Ekdahl C, Eneroth M (2007) Outcome and quality of life after surgically treated ankle fractures in patients 65 years or older. BMC Musculoskelet Disord 8: 127.

25. Hedstrom M, Ahl T, Dalen N (1994) Early postoperative ankle exercise. A study of postoperative lateral malleolar fractures. Clin Orthop 300: 193196.

26. Lehtonen H, Jarvinen TL, Honkonen S, Nyman M, Vihtonen K, et al. (2003) Use of a cast compared with a functional ankle brace after operative treatment of an ankle fracture. A prospective, randomized study. J Bone Joint Surg Am 85(2): 205-211.

27. Van Laarhoven CJ, Meeuwis JD, van der Werken C (1996) Postoperative treatment of internally fixed ankle fractures: a prospective randomised study. J Bone Joint Surg Br 78(3): 395-399.

28. Tropp H, Norlin R (1995) Ankle performance after ankle fracture: a randomized study of early mobilization. Foot Ankle 16(2): 79-83.

29. Ware JE (1996) SF-36 Health Survey: Manual and Interpretations Guide. Boston: The Health Institute. New England Medical Center 10: 13.

30. Ponzer S, Nasell H, Bergman B (1999) Functional outcome and quality of life with patients with Type B ankle fractures: a two-year follow-up study. J Orthop Trauma 13(5): 363-368.

31. Bhandari M, Sprague S, Hanson B, Busse JW, Dawe DE, et al. (2004) Health-related quality of life following operative treatment of unstable ankle fractures: a prospective observational study. J Orthop Trauma 18(6): 338-345 